\title{
Adsorption Efficiency of Synthetic Nano Iron Oxide and Commercial Activated Carbon Towards the Removal of Co(II) Ions
}

\author{
G. KANTHIMATHI ${ }^{1}$, P. KOTTEESWARAN ${ }^{2}$, \\ P. THILLAI ARASU ${ }^{1}$ and M.KOTTAISAMY ${ }^{3}$
}

${ }^{1}$ Department of Chemistry, Kalasalingam University, Krishnankoil - 626190, India

${ }^{2}$ Department of Chemistry, Ramco Institute of Technology, Rajapalayam -626117, India

${ }^{3}$ Department of Chemistry, Thiagarajar College of Engineering, Madurai - 625015, India

kanthi_somu@rediffmail.com

Received 26 August 2013 / Accepted 4 October 2013

\begin{abstract}
The synthetic nano iron oxide (SNIO) was synthesized by co-precipitation method and characterized by XRD, SEM, FT-IR and EDAX. Commercial activated carbon (CAC) was further activated by acid treatment in order to enhance the adsorption capacity. Batch adsorption experiments were carried out to compare the adsorption efficiency of SNIO and CAC towards the removal of $\mathrm{Co}$ (II) ions as a function of initial concentration, dose rate, contact time, $\mathrm{pH}$ and stirring speed. The results show that SNIO have higher adsorption capacity of $99.2 \%$ towards $\mathrm{Co}$ (II) ion whereas CAC shows only $87.8 \%$. The amount of $\mathrm{Co}(\mathrm{II})$ ion adsorbed per unit mass $\left(\mathrm{q}_{\mathrm{e}}\right)$ of SNIO was $19.31 \mathrm{mg} \mathrm{g}^{-1}$. For CAC the amount of $\mathrm{Co}(\mathrm{II})$ ion adsorbed per unit mass $\left(\mathrm{q}_{\mathrm{e}}\right)$ is only $3.38 \mathrm{mg} \mathrm{g}^{-1}$. The equilibrium adsorption isotherm data have been tested by applying both Freundlich and Langmuir adsorption isotherm. The separation factor $\mathrm{R}$ was found to be 0 to 1 for both the adsorbent; it clearly indicates feasibility of adsorption.
\end{abstract}

Keywords: Adsorption, Nano iron oxide, Equilibrium

\section{Introduction}

Higher doses of cobalt can adversely affect the hemoglobin content of the patient's blood and produce polycythemia and hyperglycemia ${ }^{1,2}$. Cobalt poisoning leads to gastrointestinal distress and heart failure. Arena 1970 also considered that cobalt causes goiters in humans. The hazardous nature of cobalt metal, even in trace quantities due to their tendency for chelation and accumulation with the organic and biomolecules makes it necessary to develop suitable cost-effective methods for the removal of heavy metals from waste water systems and industrial effluents fed into water stream. 


\section{Experimental}

Synthetic nano iron oxide (SNIO) was synthesized by co-precipitation of ferrous and ferric chloride. All the chemicals were reagent grade (SD Fine) and were used without further purification. For the preparation of nano $\mathrm{Fe}_{3} \mathrm{O}_{4}, 6.5 \mathrm{~g}$ of ferric chloride and $4.8 \mathrm{~g}$ of ferrous chloride were dissolved in $200 \mathrm{~mL}$ of distilled water and $10 \%$ polyethylene glycol together and dispersed by ultra sonic stirring for 10 minutes. Then the mixture was heated to $75^{\circ} \mathrm{C}$. The $\mathrm{pH}$ value of the mixture was adjusted to about 11.5 by using sodium hydroxide $(1 \mathrm{M})$. The mixing was continued for 2 hours at $60{ }^{\circ} \mathrm{C}$ and then agitated at $80{ }^{\circ} \mathrm{C}$ for 30 minutes, purified and washed several times with distilled water at $80{ }^{\circ} \mathrm{C}$ until the silver nitrate test shows no chloride precipitation. The chemical reaction for the precipitation of $\mathrm{Fe}_{3} \mathrm{O}_{4}$ by alkalinizing aqueous mixture of ferric and ferrous salts with $\mathrm{NaOH}$ is expected as follows ${ }^{3}$. The flow diagram and demonstration of the preparation of the nano iron oxide are given below (Figure 1).

$$
\mathrm{Fe}^{2+}+2 \mathrm{Fe}^{3+}+8 \mathrm{OH}^{-} \rightarrow \mathrm{Fe}_{3} \mathrm{O}_{4}+4 \mathrm{H}_{2} \mathrm{O}
$$

$6.5 \mathrm{~g}$ of $\mathrm{FeCl}_{3}$ and $4.8 \mathrm{~g}$ of $\mathrm{FeCl}_{2}$ dissolved in $200 \mathrm{~mL}$ of deionised water

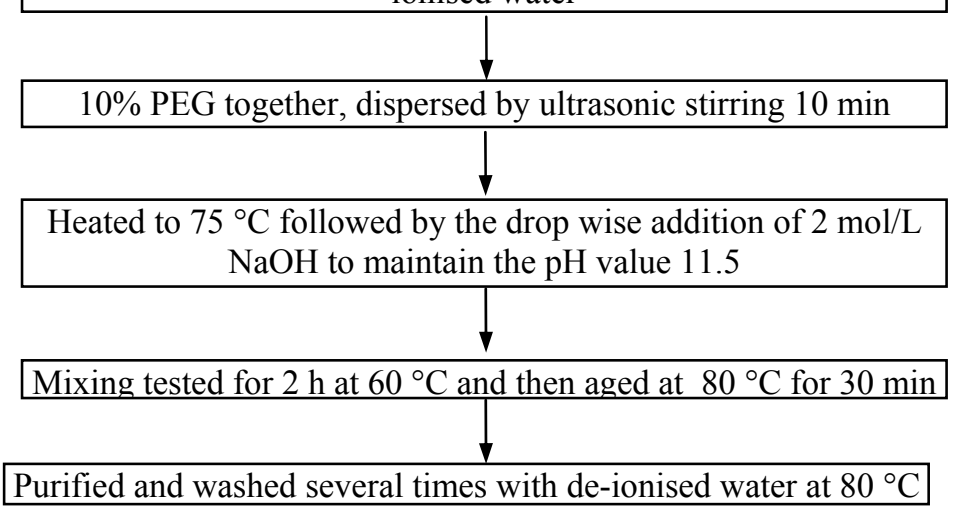

Figure 1. Demonstration of the preparation of the nano iron oxide

\section{Results and Discussion}

\section{$X$-ray analysis}

Figure $2 \mathrm{a} \& \mathrm{~b}$ shows the $\mathrm{x}$-ray diffraction pattern of the commercial micro $\mathrm{Fe}_{3} \mathrm{O}_{4}$ and chemically synthesized $\mathrm{Fe}_{3} \mathrm{O}_{4}$ particles by co-precipitation of $\mathrm{Fe}^{2+}$ and $\mathrm{Fe}^{3+}$ ions in aqueous medium. The curve shows all the diffraction peaks of the $\mathrm{Fe}_{3} \mathrm{O}_{4}$ (JCPDS-75-1609) and the broadening of the diffracted peaks of the chemically synthesized $\mathrm{Fe}_{3} \mathrm{O}_{4}$ shows a growth of nanocrystalline phase with an average size of $20 \mathrm{~nm}$ calculated from the Debye-Scherer formula.

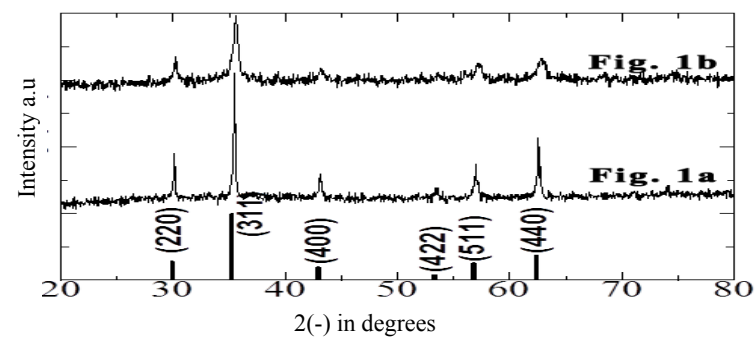

Figure 2. XRD pattern of commercial micro (a) and synthesized nano $\mathrm{Fe}_{3} \mathrm{O}_{4}$ (b) 


\section{SEM Analysis}

Figure 3 shows the high resolution scanning electron microscope (HRSEM) image of $\mathrm{Fe}_{3} \mathrm{O}_{4}$ after adsorption of $\mathrm{Co}$ (II) ions. After treating with cobalt solution, the particle are showing an increased agglomeration and surface of the particles appears to be smooth and is shown in Figure 3. A x-ray energy dispersion analysis (EDAX) of cobalt solution treated $\mathrm{Fe}_{3} \mathrm{O}_{4}$ is depicted in Figure 4. From the EDAX spectrum, it is observed that $\mathrm{Co}$ is adsorbed on the surface of $\mathrm{Fe}_{3} \mathrm{O}_{4}$.

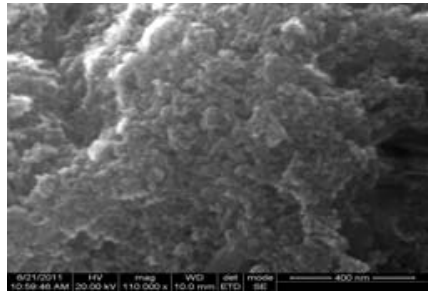

Figure 3. SEM micrograph on Cobalt adsorbed $\mathrm{Fe}_{3} \mathrm{O}_{4}$

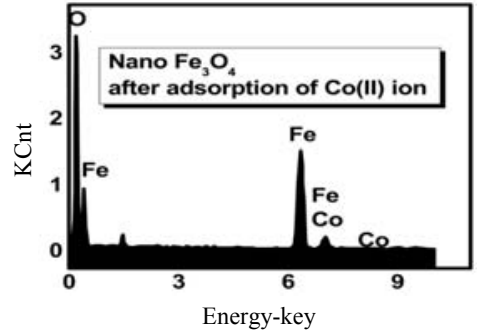

Figure 4. EDAX for Cobalt adsorbed on nano $\mathrm{Fe}_{3} \mathrm{O}_{4}$

\section{Infra-red spectroscopy (IR)}

The vibrational structure of the prepared nano materials were characterized by using Shimadzu 8400 S FTIR spectrophotometer. The IR spectra are reproduced in Figure 5. The absorption bands which are exclusively due to certain group in the prepared $\mathrm{Fe}_{3} \mathrm{O}_{4}$ and broad band at $3425 \mathrm{~cm}^{-1}$, weak bands at 2085, 1635 and $573 \mathrm{~cm}^{-1}$. The broad band around $3425 \mathrm{~cm}^{-1}$ is attributed to the hydroxyl group of water molecules adsorbed by the adsorbents. The bands around $1635 \mathrm{~cm}^{-1}$ are due to $\mathrm{C}=\mathrm{C}$ stretching vibrations ${ }^{3}$. The bands around $1580 \mathrm{~cm}^{-1}$ may be due to $\mathrm{C}-\mathrm{H}$ stretching vibrations. The band at $573 \mathrm{~cm}^{-1}$ are due to the stretching frequency of $\mathrm{Fe}-\mathrm{O}$ in nano $\mathrm{Fe}_{3} \mathrm{O}_{4}$ respectively ${ }^{3}$. They are characteristically pronounced for all spinal structures and for ferrite in particular.

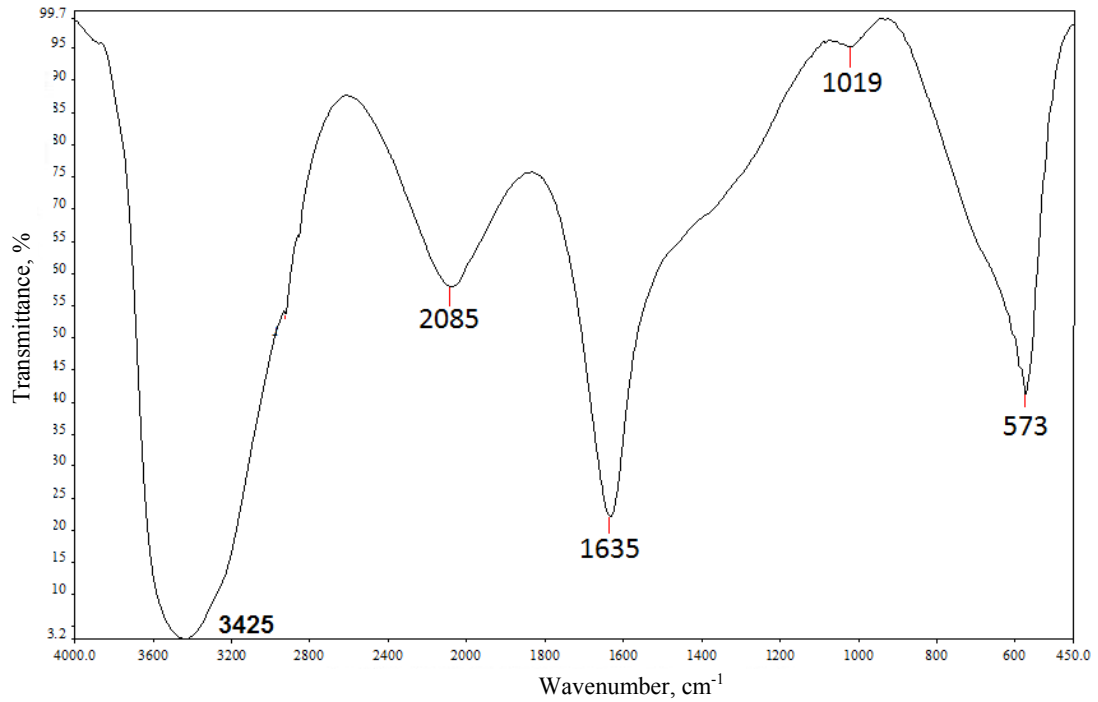

Figure 5. Infra -red spectrum of synthetic nano $\mathrm{Fe}_{3} \mathrm{O}_{4}$ 


\section{Effect of initial concentration}

Adsorption studies of $\mathrm{Co}(\mathrm{II})$ ions on SNIO and CAC at a fixed dose of adsorbent $(2.57 \mathrm{~g} / \mathrm{L}$ for SNIO and $13.3 \mathrm{~g} / \mathrm{L}$ for CAC) at different initial concentration of metal ions (range : 25 - $350 \mathrm{ppm})$ and constant contact time $(60 \mathrm{~min})$ and at solution $\mathrm{pH} \mathrm{5-6}$ for both the adsorbent at a temperature of $28 \pm 1{ }^{\circ} \mathrm{C}$ were carried out. The variations in the percentage removal of $\mathrm{Co}$ (II) ions with its concentration are shown diagrammatically in Figure 6. In both cases of adsorbents, it was observed that the percentage removal of Co(II) ions is low at higher concentration and gradually increases as the concentration of $\mathrm{Co}$ (II) ions decreases. This is due to the fact that after the formation of mono-ionic layer at lower concentration over the adsorbent surface, further formation of the layer is highly hindered at higher concentration due to the interaction between Co(II) ions present on the surface and in the solution. In addition to that, at low concentration of the Co(II) ions, the ratio of the initial number of moles of the $\mathrm{Co}$ (II) ions to the available surface area of the adsorbent is large and subsequently, the fraction of the adsorption becomes independent of the initial concentration of the metal ion. But at higher concentration, the adsorption sites available for the adsorption become lesser and hence, the percentage removal of the metal ions at higher concentration decreases. The optimum concentration of the $\mathrm{Co}$ (II) ions is found to be $50 \mathrm{ppm}$ for its efficient removal by adsorption on the SNIO. For CAC the optimum concentration of the $\mathrm{Co}$ (II) ion is found to be $51.3 \mathrm{ppm}$. The percentage removal of the $\mathrm{Co}$ (II) ions by SNIO at $50 \mathrm{ppm}$ level is found to be $99.2 \%$, whereas the CAC removes only $87.8 \%$ at $51.3 \mathrm{ppm}$ concentration of the Co(II) ions. The higher adsorptive power of the SNIO over the CAC may be attributed to the nanosize of the SNIO. When a particle decreases to the nanometer range, an increasing fraction of the atoms are exposed to the surface, giving rise to excess surface energy compared to macro molecules. Therefore, nanoparticles with a higher total energy should be prone to adsorb molecules onto their surfaces in order to decrease the total free energy ${ }^{4}$.

\section{Effect of dose rate}

The adsorption data for the removal of Co(II) ions by adsorption on SNIO and CAC with different doses of adsorbents (range: 0.25-3.57 for SNIO and 2.66-18.6 for CAC) at optimum initial concentration of $\mathrm{Co}$ (II) ions at constant contact time $(60 \mathrm{~min})$ and constant initial $\mathrm{pH}$ (5-6). The effects of dose of adsorbent on the adsorption of metal ions are represented in Figure 7 respectively for SNIO and CAC. It is noted that the percentage removal of the $\mathrm{Co}$ (II) ions increases as the adsorbent concentration increases owing to the enhanced total surface area of the adsorbent. The removal of the $\mathrm{Co}$ (II) ions increases slightly with increase in the dose of the SNIO and CAC.

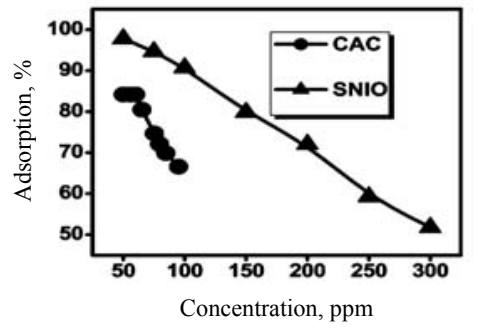

Figure 6. Effect of concentration on the percentage removal of the $\mathrm{Co}$ (II) ions by adsorption on CAC and SNIO

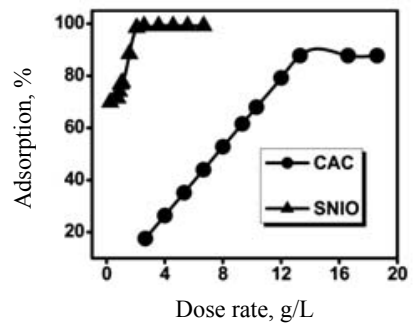

Figure 7. Effect of the dose rate in the percentage removal of the $\mathrm{Co}$ (II) ions by adsorption on SNIO and CAC 
This means that the toxic ions can be removed effectively from the contaminated water with the proper amount of the adsorbent, which would possess more adsorption sites available for the metal ion uptake from the solution ${ }^{5}$. The SNIO shows higher adsorption capacity $(99.2 \%)$ with the optimum dose of $2.57 \mathrm{~g} / \mathrm{L}$ which is nearly six times less than the CAC $(87.8 \%$ at $13.3 \mathrm{~g} / \mathrm{L})$, because the nanoparticles possess more number of micropores and mesopores and higher total surface energy than macroparticles which are more prone to adsorb the $\mathrm{Co}$ (II) ions on to the surface of the adsorbent in order to decrease the total free energy ${ }^{4}$.

\section{Effect of contact time}

In order to study the effect of the contact time on the removal of the Co(II) ions, the adsorption experiments were carried out at different contact times at optimum concentrations of the metal ion with the respective dose of the SNIO and CAC. The relevant experimental data are graphically represented in Figure 8. The percentage removal was found to be rapid at the initial period of contact time and then became slow and stagnated with increase in contact time. The equilibrium time for the maximum removal of the Co(II) ions is found to be $10 \mathrm{~min}$ for SNIO with the effective adsorption of $99.2 \%$, for CAC, 60 min with the effective adsorption of $87.8 \%$. For SNIO the optimum contact time is found to be 10 minutes for the effective removal (99.2\%) of $\mathrm{Co}(\mathrm{II})$ ions, whereas for the CAC the optimum contact time is 60 minutes for the removal of $87.8 \%$ of the $\mathrm{Co}$ (II) ions. Within 10 minutes the SNIO shows higher adsorptive power than the CAC because the former possesses large surface area $\left(63.87 \mathrm{~m}^{2} / \mathrm{g}\right)$, whereas the latter possesses small surface area $\left(1.37 \mathrm{~m}^{2} / \mathrm{g}\right)^{4}$.

\section{Effect of $p H$}

The adsorption potential of the SNIO and CAC is found at various $\mathrm{pH}$ values, keeping the system at the optimum conditions of the initial concentration of the $\mathrm{Co}$ (II) ions, adsorbent dose and contact time. The relevant experimental data are graphically represented in Figure 9. The removal of the Co(II) ions through adsorption by the SNIO and CAC is effective in slightly acidic medium and the optimum $\mathrm{pH}$ range observed for SNIO is 6.03 and CAC 5.4. At optimum $\mathrm{pH}$ (6.03 for SNIO, 5.4 for CAC) the concentration of the $\mathrm{H}^{+}$ions is lowered and consequently, the adsorption of the $\mathrm{Co}(\mathrm{II})$ ions increases. At higher $\mathrm{pH}$ than the optimum, the $\mathrm{OH}^{-}$ion concentration is increased and preferentially adsorbed on the adsorbent and the surface of the adsorbent becomes negatively charged. Moreover at higher $\mathrm{pH}$, the $\mathrm{Co}$ (II) ion form various complex anion, hydroxide complexes, etc. which are retarded by the negatively charged surface of the adsorbent ${ }^{4}$. At optimum $\mathrm{pH}$ the SNIO shows higher adsorption capacity of $99.2 \%$ whereas the CAC shows only $87.8 \%$.

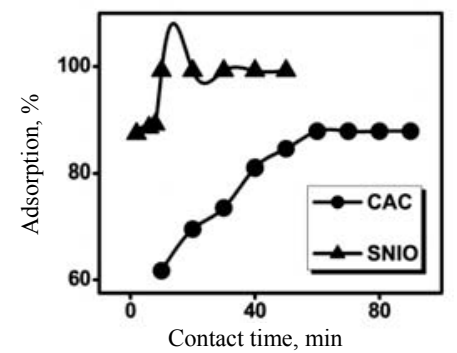

Figure 8. Effect of contact time in the percentage removal of the $\mathrm{Co}$ (II) ions by adsorption on SNIO and CAC

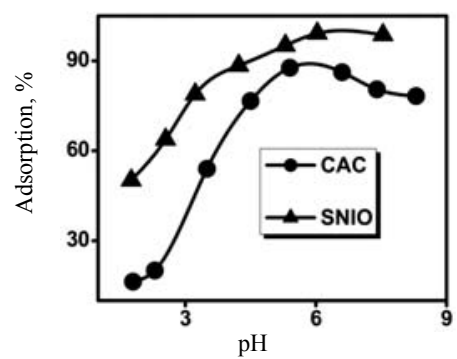

Figure 9. Variation in the percentage removal of the $\mathrm{Co}$ (II) ions by adsorption on SNIO and CAC with $\mathrm{pH}$ change. 


\section{Effect of stirring speed}

The percentage of adsorption is found to increase as the stirring speed increases and attains a steady state at a stirring speed of $135 \pm 5$. This shows that the stirring speed essentially controls the external mass transfer. The effect of the percentage removal of $\mathrm{Co}$ (II) ions by adsorption on CAC and SNIO at stirring speeds 30, 50, 70, 90, 120 $\pm 5 \mathrm{rpm}$ are represented graphically in Figure 10.

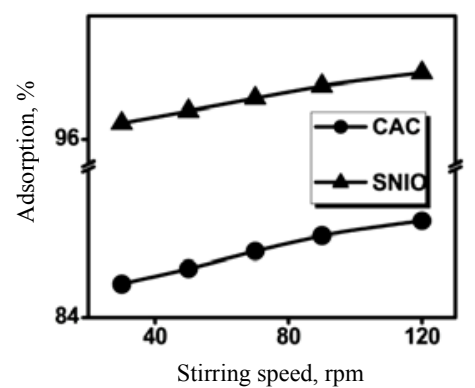

Figure 10. Variation in the percentage removal of $\mathrm{Co}(\mathrm{II})$ ions by adsorption on SNIO and $\mathrm{CAC}$ at different stirring speeds 30,50,70,90, $120 \pm 5 \mathrm{rpm}$

\section{Modeling of the adsorption}

The data obtained in the adsorption studies are analyzed by classical Freundlich and Langmuir model because the study of adsorption isotherm has been of importance and significance in the waste water treatment by the adsorption technique as they provide an approximate estimation of the adsorption capacities of the adsorbents SNIO and CAC. When the pertaining parameters for each isotherm are plotted, linear plots are observed Figure 11. The linearity indicates the applicability of all the isotherms for the Co(II) ions on the SNIO and CAC. Various useful parameters like adsorption capacities of the SNIO and CAC, energy of adsorption, separation factor and correlation co-efficient obtained from various models are collected in Table 1. Adsorption capacity is found to be high for SNIO $\left(\mathrm{Q}_{0}=333.3\right)$ than $\mathrm{CAC}\left(\mathrm{Q}_{\mathrm{o}}=4.54\right)$ and the energy of the adsorption is found to be less for SNIO $(b=0.06)$ than CAC $(b=0.3)$. The low energy of the adsorption for the SNIO may be due to the larger surface area and the highly active surface sites present in the SNIO than CAC. The separation factor R obtained for the removal of Co(II) ion by the SNIO (0.3) and the CAC (0.06) are found to be between 0 and 1 indicating the feasibility of the adsorption. The adsorption intensity $1 / \mathrm{n}$ values for the SNIO and the CAC are $>0.05$ indicating a strong bond formation between the adsorbate and the adsorbent during the adsorption.
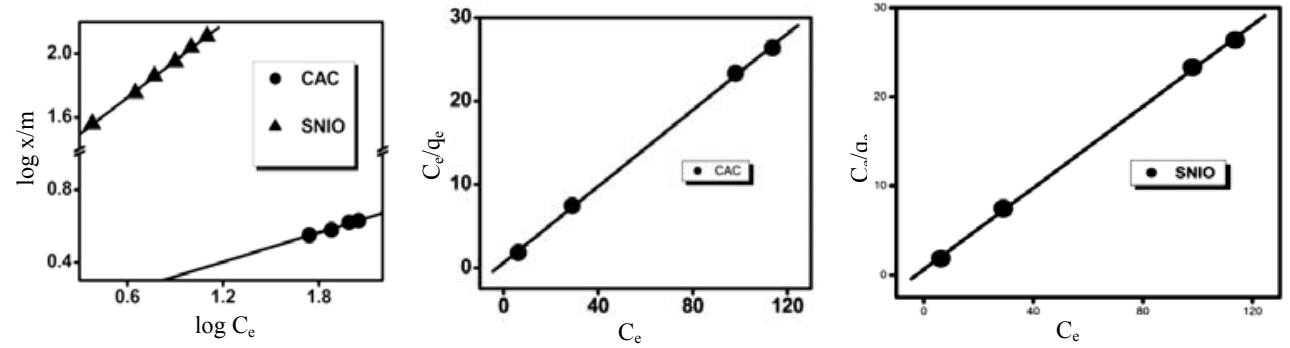

Figure 11. Application of Freundlich and Langmuir Adsorption Isotherm for the removal of the $\mathrm{Co}(\mathrm{II})$ ions adsorption on CAC and SNIO 
Table 1. Freundlich and Langmuir constants derived from the simultaneous removal of $\mathrm{Co}(\mathrm{II})$ ions by using $\mathrm{CAC}$ and $\mathrm{SNIO}$

\begin{tabular}{cccccccc}
\hline \multirow{2}{*}{ Adsorbent } & \multicolumn{3}{c}{ Freundlich constant } & \multicolumn{4}{c}{ Langmuir constant } \\
\cline { 2 - 8 } & $\mathrm{K}$ & $1 / \mathrm{n}$ & $\mathrm{r}^{2}$ & $\mathrm{Q}_{0} \mathrm{mgg}^{-1}$ & $\mathrm{~B} \mathrm{Lmg}^{-1}$ & $\mathrm{R}$ & $\mathrm{r}^{2}$ \\
\hline CAC & 1.20 & 0.26 & 0.99 & 4.54 & 0.3 & 0.06 & 0.99 \\
SNIO & 18.12 & 0.7 & 0.99 & 333.3 & 0.06 & 0.3 & 0.99 \\
\hline
\end{tabular}

\section{Conclusion}

In the present study, the efficiency of the SNIO towards the removal of the Co(II) ion was examined and compared with that of the CAC. The optimum conditions of the various factors for the maximum removal of the $\mathrm{Co}$ (II) ion arrived at from the studies are given in Table 2. The energy of the adsorption for the SNIO was found to be lower compared to the CAC. The higher adsorption capacity and lower energy of the adsorption of the SNIO compared to the CAC attributed to the large surface area and higher active surface sites of the SNIO. Hence, the SNIO may be considered to be superior, more effective and efficient alternative adsorbent than for the removal of the $\mathrm{Co}$ (II) ion than CAC.

Table 2. The optimum conditions of the various factors for the maximum removal of the $\mathrm{Co}$ (II) ions arrived at from the studies

\begin{tabular}{lcc}
\hline Parameter & SNIO & CAC \\
\hline Initial concentration of the Co(II)ion, ppm & 50 & 51.3 \\
Dose rate, g/L & 2.57 & 13.3 \\
Contact time, min & 10 & 60 \\
pH & 5.4 & 6.03 \\
Stirring Speed. rpm & $135 \pm 5$ & $135 \pm 5$ \\
Percentage Removal, $\%$ & 99.2 & 87.8 \\
Adsorption capacity, $\mathrm{mg} / \mathrm{g}$ & 333.3 & 4.54 \\
Energy of adsorption, $\mathrm{L} / \mathrm{mg}$ & 0.06 & 0.3 \\
Amount adsorbed per unit mass of the adsorbent $\left(\mathrm{q}_{\mathrm{e}}\right) \mathrm{mg} \mathrm{g}^{-1}$ & 19.31 & 3.38 \\
\hline
\end{tabular}

\section{References}

1. Davis J E and Fields J P, Fed Proc., 1955, 14, 331-332.

2. Arena J M, "Poisoning", $2^{\text {nd }}$ Edn., Springfield, Ill., Thomas, 1970, 1-715.

3. Shen Y F, Tang J, Nie Z H, Wang Y D, Ren Y and Zuo L, Sep Purif Technol., 2009, 68, 312-319; DOI:10.1016/j.seppur.2009.05.020

4. Prodan D, Chanéac C, Tronc E, Jolivet J P, Cherkaour R, Ezzir A, Noguès M and Dormann J L, J Magn Magn Mater., 1999, 203(1-3), 63-65;

DOI:10.1016/S0304-8853(99)00189-4

5. Panda L, Das B, Rao D S and Mishra B K, J Hazard Mater., 2011, 192(2), 822-831; DOI:10.1016/j.jhazmat.2011.05.098 